\title{
Hepatokines: unlocking the multi-organ network in metabolic diseases
}

\author{
Alison Iroz ${ }^{1,2,3}$ • Jean-Pierre Couty ${ }^{1,2,3,4}$ - Catherine Postic ${ }^{1,2,3}$
}

Received: 19 February 2015 / Accepted: 27 April 2015 / Published online: 2 June 2015

(C) Springer-Verlag Berlin Heidelberg 2015

\begin{abstract}
In the face of urbanisation, surplus energy intake, sedentary habits and obesity, type 2 diabetes has developed into a major health concern worldwide. Commonly overlooked in contemporary obesity research, the liver is emerging as a central regulator of whole body energy homeostasis. Liverderived proteins known as hepatokines are now considered attractive targets for the development of novel type 2 diabetes treatments. This commentary presents examples of three leading hepatokines: fetuin-A, the first to be described and correlated with increased inflammation and insulin resistance; angiopoietin-like protein (ANGPTL)8/betatrophin, initially proposed for its action on beta cell proliferation, although this effect has recently been brought into question; and fibroblast growth factor 21 (FGF21), an insulin-sensitising hormone that is an appealing drug target because of its beneficial metabolic actions. Novel discoveries in hepatokine research may lead to promising biomarkers and treatments for metabolic disorders and type 2 diabetes. This is one of a series of commentaries under the banner ' 50 years forward', giving personal opinions on future perspectives in diabetes, to celebrate the 50th anniversary of Diabetologia (1965-2015).
\end{abstract}

Keywords Beta cell · Glucose homeostasis $\cdot$ Hepatokines · Liver $\cdot$ Metabolic diseases $\cdot$ NAFLD $\cdot$ Type 2 diabetes

Catherine Postic

catherine.postic@inserm.fr

1 Inserm U1016 Institut Cochin, 24 rue du Faubourg Saint Jacques, 75014 Paris, France

2 CNRS UMR 8104, Paris, France

3 Université Paris Descartes, Sorbonne Paris Cité, Paris, France

4 Université Paris Diderot, UFR des Sciences du Vivant (SDV), Sorbonne Paris Cité, Paris, France

\begin{abstract}
Abbreviations
ANGPTL Angiopoietin-like protein

FGF21 Fibroblast growth factor 21

LECT2 Leucocyte cell-derived chemotaxin 2

LIRKO Liver-specific insulin receptor knockout

NAFLD Non-alcoholic fatty liver disease

NASH Non-alcoholic steatohepatitis
\end{abstract}

\section{Introduction}

The metabolic syndrome is a leading health concern in the wake of urbanisation, surplus energy intake, obesity and sedentary habits. It is defined by a constellation of interconnected physiological and biochemical factors that increase the risk of cardiovascular disease and type 2 diabetes, namely, insulin resistance, visceral adiposity, dyslipidaemia, fatty liver, endothelial dysfunction and elevated blood pressure. Hyperglycaemia in type 2 diabetes is the result of impaired insulin secretion and increased insulin resistance in target tissues (liver, muscle, adipose tissue). Interestingly, several proteins that are exclusively or predominantly secreted by the liver are now known to directly affect energy metabolism. Analogous to the adipokines (from adipose tissue) and myokines (from skeletal muscle), these liver-derived proteins, named hepatokines, have ambivalent roles, either promoting insulin resistance or improving metabolic variables of type 2 diabetes [1]. For example, fetuin-A, plays a major pathogenic role in metabolic diseases, while fibroblast growth factor 21 (FGF21) has recently emerged as a novel hormone, leading to beneficial effects on glucose metabolism and lipid homeostasis, in addition to promoting rapid body weight loss in rodents [2]. Several FGF21 benefits have translated to non-human primates and obese type 2 diabetic patients [3]. 
From an historical point of view, and in order to illustrate the roles of hepatokines in whole system biology, we will briefly review the current knowledge on three major hepatokines: (1) fetuin-A; (2) angiopoietin-like protein (ANGPTL)8, also known as betatrophin; and (3) FGF21. Keeping in mind the spirit of the task at hand, we will also speculate on how hepatokines may in the future represent novel biomarkers and therapeutic approaches for metabolic disorders and type 2 diabetes.

While our environment in Western societies has played a major role in the exponential growth of metabolic-related diseases, the notion that the sole cause of obesity is behaviour was first challenged in 1969 by the results of the parabiosis studies conducted by Douglas Coleman [4]. The experiments involved mice with two distinct diabetic mutations, $o b / o b$ mice, which display moderate glucose intolerance and islet hypertrophy, and $d b / d b$ mice, characterised by a shortened life expectancy and islet apoptosis. When paired to a wild-type mouse, a significant food intake-limiting effect was reported on the $o b / o b$ partner; conversely, the $d b / d b$-wild-type pairing induced severe feeding apathy in the wild-type, leading to its starvation and death [4]. Coleman concluded that the observations were the result of a circulatory satiety factor, with the $o b / o b$ mouse producing insufficient factor to regulate its food intake and the $d b / d b$ mouse producing factor that it was unresponsive to. These findings not only provided the first clues to a genetic component of obesity but also - of particular relevance to this commentary-opened a new role for organ crosstalk in the control of energy homeostasis. While Coleman's work revitalised the importance of circulatory factors in the field of diabetes, the theory of diabetes as a consequence of multi-organ dysfunction was first established during the mid-19th century. Claude Bernard, professor and physiologist at the College of France in Paris (1855-1878) founded the theory of internal secretion and first illustrated diabetes mellitus as a multi-organ disorder. His discovery of endogenous glucose production by the liver highlighted the critical role of this organ in glucose homeostasis and diabetes. Development of the liver-specific insulin receptor knockout mouse (LIRKO) by Ronald Kahn's laboratory in 2000 provided the first opportunity to explore the consequences of selective hepatic insulin resistance on systematic glucose tolerance [5]. From the results of classic physiological experiments such as parabiosis and newly developed genetic mouse models, the role of hepatokines can be unlocked.

\section{Fetuin-A}

The first liver-derived factor suggested to regulate metabolic balance through integrated organ crosstalk was fetuin-A, a $64 \mathrm{kDa}$ glycoprotein encoded by the Ahsg gene. Circulating levels of fetuin-A are increased in obesity and related disorders such as the metabolic syndrome and type 2 diabetes. Fetuin-A concentrations are tightly correlated with impaired insulin sensitivity and glucose intolerance as it directly inhibits the downstream phosphorylation events of the insulin signalling cascade and the translocation of the glucose transporter GLUT4 in insulin target tissues. Interestingly, this liverderived protein is also associated with fatty liver and correlates with non-alcoholic fatty liver disease (NAFLD) in humans [1]. Identified as an adaptor protein for saturated fatty acidmediated activation of Toll-like receptor 4, this fetuin-A-fatty acid synergy may induce inflammatory signalling and insulin resistance - two important driving forces behind type 2 diabetes [6]. Pioglitazone, one of the thiazolidinedione class of type 2 diabetes agents, significantly reduces serum fetuin-A levels in patients with type 2 diabetes. However, other insulinsensitising therapies, such as metformin and aerobic exercise, do not seem to affect fetuin-A levels [7]. Although its regulation is still largely unknown, fetuin-A still represents an attractive target for the development of type 2 diabetes treatments.

\section{ANGPTL8/betatrophin}

Since the discovery of a glucose-lowering agent in pancreatic extracts by Banting and Best in 1922 [8], improving pancreatic function, particularly endogenous insulin production by beta cells, has been the focus of pharmaceutical research. The notion of a hepatokine that could improve beta cell proliferation was based on the findings of a parabiosis study in which LIRKO mice were paired with lean controls [9]. Control partners of LIRKO mice showed increased plasma insulin concentrations associated with beta cell proliferation. Importantly, serum from LIRKO mice increased beta cell proliferation in cultured human islets, indicating that the secretome of an insulin-resistant liver is able to induce physiological changes in beta cell function/mass [9]. However, despite extensive analysis of known circulating factors, a potential candidate was not identified. Shortly following the description of liverderived secretory proteins secretion in LIRKO mice, a separate team proposed a potential candidate, ANGPTL8, renaming it betatrophin [10]. Through the use of the insulin receptor antagonist S961, the authors credited this hepatokine for the increased beta cell proliferation and mass observed under conditions of whole body insulin resistance. While direct stimulation of mouse beta cells with S961 in vitro had no effect on morphology, systemic treatment strikingly increased beta cell proliferation and mass. Initially promising, the role of ANGPTL8 as a hepatokine controlling beta cell growth has been challenged. Angptl8 knockout mice, while exhibiting profoundly reduced triacylglycerol levels, show no abnormalities in terms of glucose homeostasis and/or in beta cell expansion in response to insulin resistance resulting from either a high-fat diet or from the administration of S961 [11]. 
Overexpression of ANGPTL8 in the liver of mice did not affect beta cell expansion [12]. Importantly, ANGPTL8 was reported to be increased in serum of type 1 diabetic patients, suggesting that hepatic insulin resistance is not necessary for ANGPTL 8 release. In addition, despite increased serum ANGPTL8 concentrations, ANGPTL8 was unable to rescue the massive beta cell apoptosis characteristic of type 1 diabetic patients [13]. Regardless of current criticism, the fact that plasma triacylglycerol levels seem to be correlated with ANGPTL8 expression in mice supports the possibility that inhibiting ANGPTL8 may represent an efficient therapeutic strategy for the prevention of hypertriacylglycerolaemia.

\section{FGF21}

FGF21 has been noted as a promising pharmacological target because of its ability to reduce blood glucose independently of insulin by increasing the expression of glucose transporter GLUT1 in human primary adipocytes [2]. In addition, FGF21 decreases circulating triacylglycerol levels by reducing lipolysis and increasing $\beta$-oxidation in the liver and adipose tissue [3]. Classically observed to be upregulated by the nuclear receptor peroxisome proliferator-activated receptor $\alpha$ $(\operatorname{PPAR} \alpha)$ during fasting [14], recent studies have demonstrated that FGF21 is also upregulated in hepatocytes stimulated by high glucose and that its increased expression is directly induced by the glucose-sensitive transcription factor known as carbohydrate responsive element-binding protein (ChREBP) [15]. This suggests an unexpected role for FGF21 in the response to glucose overload. A cohort of both lean adults and adults with the metabolic syndrome experienced a marked increase in plasma levels of FGF21 60 min after a fructose and glucose bolus [16], making FGF21 the first hepatokine to be identified as being regulated by nutritional status. In addition, hepatic expression and circulating concentrations of FGF21 are increased in patients with NAFLD [17]. These observations point to a central role of FGF21 in metabolic regulation and suggest that carbohydrate-induced FGF21 secretion may be instrumental in preventing nutritional-induced lipotoxicity by partitioning excess nutrients. In line with this, mice deficient in hepatic glucose 6phosphatase (a key enzyme in glucose production) fed a high-fat/high-sucrose diet, are resistant to obesity and type 2 diabetes, despite the onset of hepatic steatosis, owing to the secretion of liver-derived proteins such as

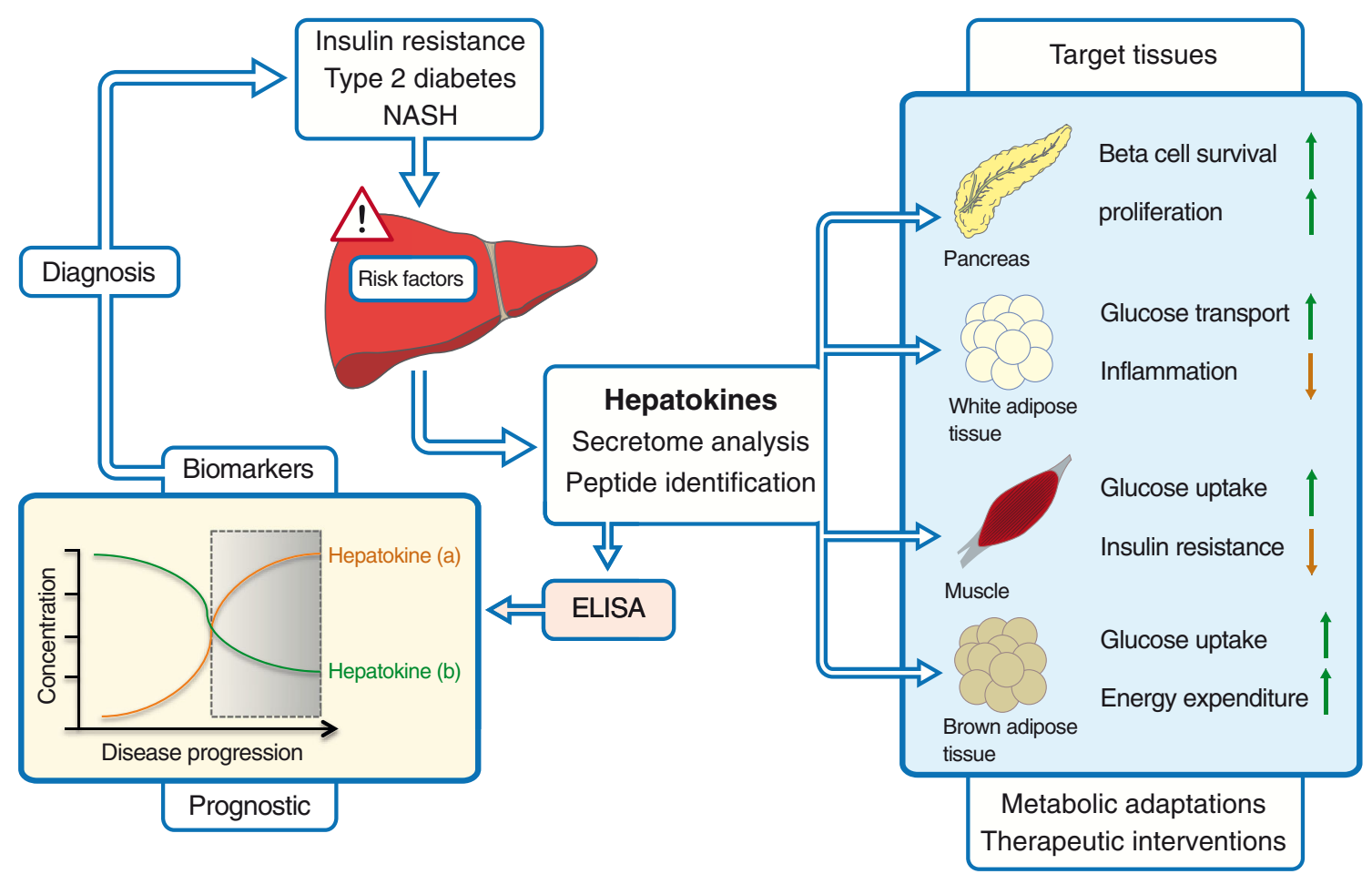

Fig. 1 Hepatokines as biomarkers and therapeutic approaches for metabolic diseases. The liver plays a central role in orchestrating whole body energy metabolism through the secretion of hepatokine(s) in response to stress signals (insulin resistance, type 2 diabetes, NASH). Combining proteomic techniques and bioinformatics software platforms may identify variations in pathogenic or beneficial hepatokine(s) (lines a and b) as potential biomarkers for the progression of diseases such as type 2 diabetes and/or NASH. Ideally, hepatokine discovery would also lead to the development of therapeutics to control energy homeostasis, insulin sensitivity and glucose uptake in peripheral tissues, while reducing resistance and inflammation in target tissues. Improvement of specific tissue function could then reduce the pancreatic workload and normalise hepatic glucose production 
FGF21 and ANGPTL6 and the subsequent activation of peripheral glucose metabolism and thermogenesis [18].

\section{Hepatokine potential}

Evaluation of insulin resistance (or sensitivity) and beta cell function is key to understanding diabetes progression and for decision-making regarding the choice of pharmacological treatment(s). The gold standard method for assessment of insulin sensitivity is the hyperinsulinaemic-euglycaemic clamp, but this approach remains costly and time consuming and is therefore mostly limited to clinical research. HOMA-IR, calculated from fasting plasma glucose level and immunoreactive insulin, is, in contrast to the glucose clamp, a simple method for assessing insulin sensitivity. However, HOMAIR is not always reliable and is difficult to apply to patients with poor glycaemic control, severe beta cell dysfunction and/ or receiving insulin therapy. In this context, hepatokines may have the potential to provide simple and effective strategies for disease detection/progression and patient care, and may serve as therapeutic targets for various metabolic diseases, including type 2 diabetes (Fig. 1).

Another field of interest is NAFLD, the hepatic component of the metabolic syndrome. NAFLD ranges from simple benign steatosis to non-alcoholic steatohepatitis (NASH). Distinguishing simple steatosis from NASH is crucial, since the latter can progress to end-stage liver disease, like fibrosis, cirrhosis and hepatocellular carcinoma. Liver biopsy, considered the gold standard for NASH diagnosis, is invasive and costly. Imaging studies (ultrasonography or magnetic resonance imaging) have limited sensitivity for steatosis detection and are currently not able to distinguish steatosis from NASH. Therefore, reliable non-invasive biomarkers are needed for NASH patients, to establish diagnosis, follow disease progression and implement efficient therapeutic strategies. Proteomic techniques combined with mass spectrometry and bioinformatics software should help to identify and quantify "networking' proteins in the secretome of patients at risk for type 2 diabetes or NASH (Fig. 1). In this context, one potential interesting lead is leucocyte cell-derived chemotaxin 2 (LECT2), a secreted molecule mainly produced by hepatocytes that exerts an anti-inflammatory effect in $\beta$-catenininduced hepatic carcinoma [19]. According to recent observations in a cohort of type 2 diabetic patients, circulating levels of LECT2 positively correlate with BMI and hepatic steatosis [20]. These data suggest that LECT2 could be a new player in the mechanisms of insulin resistance and may constitute a biomarker for patient follow-up, notably in those at risk for developing type 2 diabetes. However, circadian rhythms need to be taken into account before considering a liver-secreted protein as a potential biomarker. In particular, the fact that baseline concentrations of circulating FGF21 are driven by circadian control and that the early morning FGF21 peak observed in lean individuals is completely lost in obese patients [21], highlights the importance of fully characterising the circadian relationship of future hepatokines in order to determine their suitability as potential biomarkers.

From a therapeutic point of view, LY2405319, an FGF21 analogue that is already in clinical trial, has shown a significant and positive impact on reducing body weight and fasting insulin in obese and diabetic patients after only 28 days of daily subcutaneous injections [22]. Over the study period, although glycaemia only showed a trend towards a decrease, body weight was significantly reduced. These results could be promising since weight reduction has substantial beneficial effects on the metabolic syndrome. A recent publication using insulin-deficient diabetic mice (resulting from streptozotocin treatment) reported the importance of multiple tissue networking in regulating blood glucose [23]. Improvement of hyperglycaemia following LY2405319 treatment was due to the utilisation of glucose by brown adipose tissue through upregulation of the glucose transporters GLUT1 and GLUT4. Removal of brown adipose tissue obliterated the glucose-lowering effect of LY2405319 [23]. Personalised therapies that could specifically target glucose utilisation in peripheral tissues (adipose tissue, muscle) could greatly improve the quality of life of patients by reducing the need for insulin injections. A better understanding of the molecular basis of FGF21 expression in the liver represents a necessary step for efficient therapeutic approaches.

Acknowledgements The authors would like to thank S. Guilmeau and J. Girard (Institut Cochin Inserm U1016) for helpful comments and critical reading of the manuscript.

Contribution statement All authors were responsible for drafting the article and revising it critically for important intellectual content. All authors approved this version to be published.

\section{References}

1. Stefan N, Haring HU (2013) The role of hepatokines in metabolism. Nat Rev Endocrinol 9:144-152

2. Kharitonenkov A, Adams AC (2014) Inventing new medicines: the FGF21 story. Mol Metab 3:221-229

3. Gimeno RE, Moller DE (2014) FGF21-based pharmacotherapypotential utility for metabolic disorders. Trends Endocrinol Metab 25:303-311

4. Coleman DL (1973) Effects of parabiosis of obese with diabetes and normal mice. Diabetologia 9:294-298

5. Michael MD, Kulkarni RN, Postic C et al (2000) Loss of insulin signaling in hepatocytes leads to severe insulin resistance and progressive hepatic dysfunction. Mol Cell 6:87-97 
6. Pal D, Dasgupta S, Kundu R et al (2012) Fetuin-A acts as an endogenous ligand of TLR4 to promote lipid-induced insulin resistance. Nat Med 18:1279-1285

7. Ochi A, Mori K, Emoto M et al (2014) Direct inhibitory effects of pioglitazone on hepatic fetuin-A expression. PLoS One 9, e88704

8. Banting FG, Best CH (1922) The internal secretion of the pancreas. J Lab Clin Med 7:251-266

9. El Ouaamari A, Kawamori D, Dirice E et al (2013) Liver-derived systemic factors drive beta cell hyperplasia in insulin-resistant states. Cell Rep 3:401-410

10. Yi P, Park JS, Melton DA (2013) Betatrophin: a hormone that controls pancreatic beta cell proliferation. Cell 153:747-758

11. Gusarova V, Alexa CA, Na E et al (2014) ANGPTL8/betatrophin does not control pancreatic beta cell expansion. Cell 159:691-696

12. Cox AR, Lam CJ, Bonnyman CW, Chavez J, Rios JS, Kushner JA (2015) Angiopoietin-like protein 8 (ANGPTL8)/betatrophin overexpression does not increase beta cell proliferation in mice. Diabetologia. doi:10.1007/s00125-015-3590-z

13. Espes D, Lau J, Carlsson PO (2014) Increased circulating levels of betatrophin in individuals with long-standing type 1 diabetes. Diabetologia 57:50-53

14. Badman MK, Pissios P, Kennedy AR, Koukos G, Flier JS, MaratosFlier E (2007) Hepatic fibroblast growth factor 21 is regulated by PPAR $\alpha$ and is a key mediator of hepatic lipid metabolism in ketotic states. Cell Metab 5:426-437

15. Iizuka K, Takeda J, Horikawa Y (2009) Glucose induces FGF21 mRNA expression through ChREBP activation in rat hepatocytes. FEBS Lett 583:2882-2886
16. Dushay JR, Toschi E, Mitten EK, Fisher FM, Herman MA, Maratos-Flier E (2014) Fructose ingestion acutely stimulates circulating FGF21 levels in humans. Mol Metab 4:51-57

17. Dushay J, Chui PC, Gopalakrishnan GS et al (2010) Increased fibroblast growth factor 21 in obesity and nonalcoholic fatty liver disease. Gastroenterology 139:456-463

18. Abdul-Wahed A, Gautier-Stein A, Casteras S et al (2014) A link between hepatic glucose production and peripheral energy metabolism via hepatokines. Mol Metab 3:531-543

19. Anson M, Crain-Denoyelle AM, Baud V et al (2012) Oncogenic $\beta$ catenin triggers an inflammatory response that determines the aggressiveness of hepatocellular carcinoma in mice. J Clin Invest 122: 586-599

20. Okumura A, Unoki-Kubota H, Matsushita $Y$ et al (2013) Increased serum leukocyte cell-derived chemotaxin 2 (LECT2) levels in obesity and fatty liver. Biosci Trends 7:276-283

21. Yu H, Xia F, Lam KS et al (2011) Circadian rhythm of circulating fibroblast growth factor 21 is related to diurnal changes in fatty acids in humans. Clin Chem 57:691-700

22. Gaich G, Chien JY, Fu H et al (2013) The effects of LY2405319, an FGF21 analog, in obese human subjects with type 2 diabetes. Cell Metab 18:333-340

23. Kim JH, Bae KH, Choi YK et al (2015) Fibroblast growth factor 21 analogue LY2405319 lowers blood glucose in streptozotocin-induced insulin-deficient diabetic mice by restoring brown adipose tissue function. Diabetes Obes Metab 17:161-169 